\title{
Eliminating the substitution axiom from Unity logic
}

\section{Report}

Author(s):

Sanders, Beverly A.

Publication date:

1990

Permanent link:

https://doi.org/10.3929/ethz-a-000548955

Rights / license:

In Copyright - Non-Commercial Use Permitted

Originally published in:

ETH, Eidgenössische Technische Hochschule Zürich, Departement Informatik, Institut für Computersysteme 128 
ETH

Eidgenössische

Technische Hochschule

Zürich
Departement Informatik Institut für

Computersysteme

Beverly Sanders

Eliminating the Substitution Axiom from UNITY Logic

May 1990 
Authors' address:

Computersysteme ETH-Zentrum

$\mathrm{CH}-8092$ Zurich, Switzerland

e-mail: sanders@inf.ethz.ch

(C) 1990 Departement Informatik, ETH Zürich 


\begin{abstract}
The UNITY substitution axiom, "if $(x=y)$ is an invariant of a program, then $x$ can be replaced by $y$ in any property of the program," is problematic for several reasons. In this paper, dual predicate transformers sst and wst are introduced that allow the strongest invariant of a program to be expressed, and these are used to give new definitions for the temporal operators unless and ensures. With the new definitions, the substitution axiom is no longer needed, and can be replaced by an inference rule which is formally justified in the logic. One important advantage is that the effects of the initial conditions on the properties of a program are formally captured in a convenient way, and one can forget about substitution in formal treatments of the UNITY proof system while still having it available when desirable to use during the derivation of programs. Composibility and completeness of the modified logic are also discussed.
\end{abstract}

Publication note:

A preliminary draft of this paper was written on 6 February, 1990. This version has been revised incorporating several suggestions and in light of the appearance, in the meantime, of [Mi90b]. 


\section{Introduction}

The UNITY programming methodology invented by Chandy and Misra [ChMi88] comprises a programming notation and a programming logic. A program consists of variable declarations, initial conditions, and a finite set of simultaneous, conditional assignment statements. The programming logic is based on the temporal operators unless, ensures, and $\mapsto$ (read leads-to), plus invariant and the substitution axiom. Although the both the language and the logic are surprisingly simple, Chandy and Misra and others have demonstrated with a large number of examples that this method is a tractable way to formally derive a wide variety of interesting parallel algorithms. UNITY promises to become an increasingly important tool in the future.

Probably because of the informal way it is stated, together with the very reasonable sounding justification for it as a generalization of Leibniz's Rule, the substitution axiom has long been neglected and misunderstood. Adding it to the original definitions* of unless, entsures, and $\mapsto$ as is done in [ChMi88] makes the proof system unsound, a fact which was apparently not noticed until the summer of 1989 (but fortunately has few practical effects). Omitting it, as has been done in virtually all of the theoretical studies of UNITY of which 1 am aware, leaves the proof system incomplete. In [Mi90b], the definitions of unless and ensures have been revised so that the proof system with substitution axiom is not unsound, but we are still left with an informally stated axiom which is difficult to handle and who's role in the logic is obscure.

In this paper, after a brief introduction to UNITY, modifications to the logic that eliminate the need for the substitution axion are proposed. The basic idea is to use the dual predicate transformers sst and wst which allow the strongest invariant of a program to be expressed. Modified definitions unless', ensures', and $\mapsto{ }^{\prime}$ are given, as well as subscripted properties which are defined and then used in new union theorems. Finally, the completeness of the modified logic is discussed.

* when these are understood in the obvious way based on the notation and the way they are used in proofs in [ChMis8] 


\section{A short introduction to UNITY}

This section presents the most essential aspects of UNITY. For more information, and many examples using it to derive programs, see Chandy and Misra's book [ChMi88].

\subsection{Programing notation and operational interpretation}

For our purposes, a UNITY program F consists of 3 sections. The declare section, F.DECLARE is a set of variable declarations (variable names together with their types), the initially section, F.INIT, is a predicate that characterizes the allowed initial states, and the assign section, F.ASSIGN, is a non-empty set of simultaneous, conditional assignment statements. The always section is not considered in this paper since any program with a non-empty always section can be transformed to an equivalent program without one. Where no confusion can arise, the name of the program will often be omitted. An execution of a UNITY program would begin in a state satisfying INIT and repeatedly execute (atomically) statements in the assign section. The choice of the statement to execute at each step is non-deterministic and subject only to the faimess constraint that each statement is chosen infinitely often. The concept of termination is replaced with that of fixed points, i.e. a state where no statement execution will cause a state change.

\subsection{Programming logic}

\subsubsection{Notation}

For a program $\mathrm{F}$, the state space of the program is the Cartesian product of the possible values of the program variables which are determined by the declared types. I will typically use $\mathrm{P}, \mathrm{Q}, \mathrm{R}, \mathrm{I}, \mathrm{J}, \mathrm{K}$, and INIT to denote total predicates on the state space of the program. The predicate True is true in all states, the predicate False is false in all states. Universal quantification over all states will be denoted by surrounding the predicate with square brackets, (i.e. the everywhere operator from [DiSc90]) The predicate transformers wlp.s.P (weakest liberal precondition) and sp.Q.s (strongest postcondition) are defined in the usual way and thus are universally conjunctive and disjunctive, respectively. Since all statements in a UNITY program are guaranteed to terminate, for all predicates $\mathrm{P}$ and statements [wlp.s.P $=\mathbf{w p} . \mathbf{\text { s.P. }}$ ] where $w p$ is the weakest precondition. The definitions in the next section are taken from [ChMi88] or [Mi90b], except that I have replaced the Hoare triples with wlp. $(\{P\} s\{Q\}$ has been replaced by $P \Rightarrow$ wlp.s.Q.) Definitions of technical terms such as 
univerally conjunctive and statements of well-known theorems used in proofs are given in the appendix.

\subsubsection{Safety properties}

Definition (unless)

$$
P \text { unless } Q \text { in } F \equiv(\Leftarrow)\langle\forall s: s \in F . A S S I G N::[P \wedge \neg Q \Rightarrow \text { wlp.s. }(P \vee Q)]\rangle
$$

Equivalence $(\equiv)$ was used in [ChMi88], follows from $(\Leftrightarrow)$ in [Mi90]. The operational interpretation of unless is that if $\mathrm{P}$ ever becomes true during the program execution, then $P$ will remain true at least as long as $\neg Q$. stable is defined to be a special case of unless, i.e. stable $\mathrm{P} \equiv \mathrm{P}$ unless False. The operational interpretation of stable $\mathrm{P}$ is that once $\mathrm{P}$ holds during the program execution, it will hold forever. An invariant property is one that holds during the entire program execution and is defined as invariant $\mathrm{I} \equiv$ stable I $\wedge[\mathrm{INIT} \Rightarrow \mathrm{I}]$. Operationally, invariant I implies that all states satisfying $\neg \mathrm{I}$ are unreachable.

\subsubsection{Liveness properties}

The basic operator for specifying liveness properties is $\mapsto$, which is defined in terms of the operator ensures.

\section{Definition (ensures)}

P ensures $\mathrm{Q}$ in $\mathrm{F} \equiv(\Leftarrow)$

$(P$ unless $Q) \wedge\langle\exists s: s \in$ F.ASSIGN:: $[\mathrm{P} \wedge \neg Q \Rightarrow$ wlp.s. $(Q)]\rangle$

\section{Definition $(\mapsto)$}

The following 3 proof rules are provided for proving liveness properties:

(ensures promotion)

Pensures Q

$$
\mathrm{P} \mapsto \mathrm{Q}
$$

(transitivity)

$$
\mathrm{P} \mapsto \mathrm{R}, \mathrm{R} \mapsto \mathrm{Q}
$$

$$
\mathrm{P} \mapsto \mathrm{Q}
$$

(disjunction) for any set W

$$
\langle\forall \mathrm{m}: \mathrm{m} \in \mathrm{W}:: \mathrm{P}(\mathrm{m}) \mapsto \mathrm{Q}\rangle
$$

$\langle\exists m: m \in W:: P(m)\rangle \mapsto Q$ 
The operational interpretation of ensures is that if $\mathrm{P}$ holds at some point during the execution of the program, then $P$ will hold at least until Q does, Q will eventually hold, and there is a single statement in the program which is guaranteed to cause this progress. The operational interpretation of $\rightarrow$ is that if $P$ holds at some point during the execution of the program, then within a finite number of statement executions, $Q$ will hold.

\subsubsection{The substitution axiom}

A final, very important additional aspect of UNITY logic, which has been ignored in virtually every study of the UNITY proof system of which I am aware (e.g. [GePn89], [JKR89], [Kn89], [Liu89]]) is the substitution axiom. The substitution axiom is stated: "if $(x=y)$ is an invariant of a program, then $x$ can be replaced by $y$ in all properties of the program". The axiom is informally justified as a generalization of Leibniz's rule for substitution of equals. The most frequent use of the rule involves invariants: one can, for example, prove $\mathrm{P} \wedge \mathrm{I}$ unless $\mathrm{Q}$ for some invariant $\mathrm{I}$, and then use the substitution axiom with $\mathrm{I}=$ True to conclude $\mathrm{P}$ unless $\mathrm{Q}$.

It is easy to see that combining the substitution axiom with the above definitions of unless and ensures which use $\equiv$ results in an unsound proof system. Suppose invariant $\mathrm{I}$ and $\langle\forall \mathrm{s}: \mathrm{s} \in \mathrm{F}$.assign $::[\mathrm{P} \wedge \mathrm{I} \wedge \neg \mathrm{Q} \Rightarrow$ wlp.s. $((\mathrm{P} \wedge \mathrm{I}) \vee \mathrm{Q})]\rangle$. Then we may conclude $\mathrm{P} \wedge \mathrm{I}$ unless $\mathrm{Q}$ from the definition of unless, $\mathrm{P}$ unless $\mathrm{Q}$ from the substitution axiom, and $[\mathrm{P} \wedge \neg \mathrm{Q} \Rightarrow$ wlp.s. $(\mathrm{P} \vee \mathrm{Q})]$ from the definition of unless. The latter conclusion is clearly not justified. As a concrete counter-example, suppose the program has one statement $\mathrm{x}:=\mathrm{x}+\mathrm{x}$, with $\mathrm{INIT} \equiv \mathrm{x}>0$. Then we may conclude $\mathrm{x}=\mathrm{k}$ unless $\mathrm{x}>\mathrm{k}$ for all $\mathrm{k}$ using the definition of unless and the substitution axiom with invariant $\mathrm{x}>0$. Taking the next step, however, leads us to conclude for all $\mathrm{k}$, $\mathrm{x}=\mathrm{k} \Rightarrow \mathrm{x}+\mathrm{x}>\mathrm{k}$. Changing equivalence in the definition to follows from solves the problem by disallowing the final step.

Operationally, the situation can be explained by noting that, depending on the initial condition, some states may be unreachable during the execution of the program. The definition of $\mathrm{P}$ unless $\mathrm{Q}$, for example, requires $[\mathrm{P} \wedge \neg \mathrm{Q} \Rightarrow$ WLP. $(\mathrm{P} \vee \mathrm{Q})]$ which is

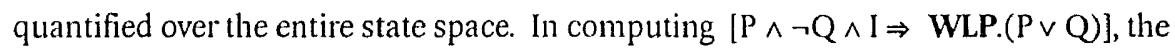
(unreachable) states where $\neg$ I holds have been eliminated from consideration. It is 
also obvious that without being able to somehow eliminate unreachable states, the proof system is incomplete. The modified logic introduced in the next section does not need the substitution axiom and is relatively complete.

\section{The modified logic}

In this section, modifications to UNITY logic that eliminate the problems described above are introduced. First, we define the predicate transformers sst and wst, then use these to give new definitions for unless and ensures, and thus also for $\mapsto$. Then, we introduce subscipted properties which are convenient for bookkeeping of the invariants that have been used in a proof and proving derived rules. The substitution axiom can then be replaced with with formally justified inference rules.

\subsection{Predicate transformers sst and wst}

It is convenient to define predicate transformers WLP and SP which apply to the entire assign section of programs.

\section{Definition (WLP and SP)}

WLP.F.P $\equiv\langle\wedge s: s \in$ F.ASSIGN :: wlp.s.P $\rangle$

SP.F.P $\equiv\langle\vee s: s \in$ F.ASSIGN ::sp.s.P $)\rangle$

Remark: WLP and SP are duals, i.e.

$\langle\forall$ predicates $P$ and $Q::[(P \Rightarrow$ WLP.F.Q $)=($ SP.F.P $\Rightarrow Q)]\rangle$. From the junctivity properties of wlp and sp: WLP is universally conjunctive and SP is universally disjunctive.

Now, we define the dual predicate transformers sst and wst (strongest and weakest "stable" predicate, respectively).

\section{Definition (sst and wst)}

(1) sst.F.P $\equiv$ strongest solution of $X:[X \Rightarrow W L P . F, X] \wedge[P \Rightarrow X]$

(2) wst. F.Q $\equiv$ weakest solution of $Y:[Y \Rightarrow$ WLP.F. $Y] \wedge[Y \Rightarrow Q]$

Obviously, both definitions could have been given in terms of SP. To simplify the notation, I will often use sst.P and wst.Q to indicate sst.F.P and wst.F.Q when this will cause no confusion. There is a difference between the word stable used in the 
sense of satisfying $[\mathrm{X} \Rightarrow$ WLP.F. $\mathrm{X}]$ and the property stable. As will be seen in the next section, stable $\mathrm{P}$ may be a property of a program even though [P $\Rightarrow$ WLP. P] does not hold. (The difference was already pointed out in [Ke76] using the term inductive for properties where $[X \Rightarrow$ WLP.F. $X]$ holds.)

The necessary results about sst and wst are given in the next theorem:

Theorem (sst and wst)

a. sst.F.P and wst.F.Q exist for every program $F$ and predicates $P$ and $Q$ and are unique.

b. $\quad$ sst and wst are duals.

c. $\quad$ sst. $\mathrm{P}=\langle\exists \mathrm{i}: 0 \leq \mathrm{i}:: \mathrm{f}$. False $\rangle$

where $\mathbf{f} . X=S P . X \vee P$

d. $\quad$ wst. $Q=\left\langle\forall \mathrm{i}: 0 \leq \mathrm{i}:: \mathrm{g}^{\mathrm{i}}\right.$.True $\rangle$

where $\mathbf{g} . \mathrm{Y}=\mathrm{WLP} . \mathrm{Y} \wedge \mathrm{Q}$

Proof:

Below, $v \mathrm{X}$ denotes the strongest solution $\mathrm{X}$, and $\mu \mathrm{X}$ the weakest solution $\mathrm{X}$.

Proof of $a$. and c. for sst

From duality of WLP and SP, plus pred. calc. (1) can be rewritten as sst.P $=v X:[(S P . X \vee P) \Rightarrow X]$

$\Rightarrow \quad\{$ SP.X $\vee P$ universally disjunctive, Knaster-Tarski $\}$

sst.P exists and is unique and sst.P $=v X:[(S P . X \vee P)=X]$,

$\Rightarrow$ \{limit theorem, SP.X $\vee$ P or-continuous $\}$

sst. $P=\langle\exists i: 0 \leq i:: \mathbf{f}$. False $\rangle$ where $\mathbf{f} X$ is defined above

Proof of a. and d. for wst

wst. $Q=\mu Y:[Y \Rightarrow$ WLP. $Y \wedge Q]$

$\Rightarrow \quad\{$ WLP.Y $\wedge$ Q universally conjunctive, Knaster-Tarski\}

wst. $Q$ exists and is unique and wst. $Q=\mu Y:[Y=W L P$. $Y \wedge Q]$

$\Rightarrow$ \{limit theorem, WLP. $Y \wedge Q$ and-continuous $\}$

wst $\mathrm{Q}=\langle\forall \mathrm{i}: 0 \leq \mathrm{i}:: \mathrm{g}$.True $\rangle$ where $\mathrm{g} . \mathrm{Y}$ is defined above

Proof of b, i.e. [sst. $P \Rightarrow Q \equiv P \Rightarrow$ wst. $Q$ ]

proof of $\Rightarrow$

sst.P $\Rightarrow Q$

$=\{$ definition of sst.P $\}$

sst. $P \Rightarrow Q \wedge P \Rightarrow$ sst.P $\wedge$ sst.P $\Rightarrow$ WLP. sst.P

$\Rightarrow$ sst.P a solution of $X:[X \Rightarrow W L P . X \wedge X \Rightarrow Q]$, but wst.Q weakest $\}$ $P \Rightarrow$ sst.P $\wedge$ sst.P $\Rightarrow$ wst.Q

$\Rightarrow$

$\mathrm{P} \Rightarrow$ wst.Q

proof of $\Leftarrow$

similar. 
wst and sst are essentially Lamport's win and sin predicate transformers [La87] which he used to extend the Owicki-Gries method (for parallel "sequential processes") to allow reasoning about programs without specifying atomic actions. Due to the UNITY setting, sst and wst have simpler recursive definitions than the corresponding results for win and sin. wst is also a special case of wsafe (i.e. [wst.P = wsafe.P.False]) defined in [JKR89] which "captures the notion of safety for a [UNITY] program". However, they did not notice that the dual of wsafe can be conveniently used to express the strongest invariant and in fact their theorem 9, which relates unless to wsafe using the definition of unless from [ChMi88], is not correct unless the substitution axiom is omitted from the logic. In any case, sst and wst are used in a completely different way than win, sin and wsafe: namely to capture the influence of the initial conditions on both the safety and liveness properties of a program.

\section{2 unless' and ensures'}

As mentioned above, we are looking for a way to allow unreachable states, which depend on the initial conditions, to be disregarded when verifying properties. In particular, we can use the fact that the sst.INIT is the strongest invariant property of a program and corresponds to the set of reachable states. Using sst and wst, we have two equivalent definitions of unless'. The new definitions are denoted with an apostrophe.

\section{Definition (unless')}

$$
\begin{aligned}
& P \text { unless' } Q \equiv[\text { sst.INIT. } \Rightarrow(P \wedge \neg Q \Rightarrow \text { WLP. }(P \vee Q))] \\
& \equiv[\text { INIT } \Rightarrow \text { wst. }(P \wedge \neg Q \Rightarrow \text { WLP. }(P \vee Q))]
\end{aligned}
$$

The equivalence of the two definitions follows from the duality of sst and wst. As before, we can define stable' and invariant' as special cases of unless': stable' $\mathrm{P} \equiv$ $\mathrm{P}$ unless' False and invariant' $\mathrm{P} \equiv$ stable' $\mathrm{P} \wedge$ [INIT $\Rightarrow \mathrm{P}$ ]. The ncw definitions are weaker than Chandy and Misra's; unless' is now completely characterized while allowing unreachable states to be disregarded. The next theorem states that any predicate implied by sst.INIT is an invariant of the program.

\section{Theorem (invariant')}

$$
[\text { sst.INIT } \Rightarrow \mathrm{P}] \equiv[\mathrm{INIT} \Rightarrow \text { wst. } \mathrm{P}] \equiv \text { invariant }^{\mathrm{P}} \mathrm{P}
$$




\section{Corollary}

invariant' True holds for all programs.

A practical advantage of the new definitions is seen when we compare the invariant theorem with the method (very similar to UNITY) independently proposed by Shankar and Lam [ShLa87] for verifying safety and liveness properties of time dependent distributed systems. In their technique, safety properties are specified by invariants. Often, one cannot verify the desired invariant I directly, but proves some stronger property, $I \wedge J$ is invariant, then concludes $I$ is invariant. They offer a heuristic to systematically generate appropriate stronger invariants. On closer examination, the heuristic is just an algorithm to compute wst.I using the computational definition of wst given above. The calculation can be terminated when gi. True $=$ True, at which point the computed invariant is in fact the weakest invariant that will do. Several properties of the heuristic can immediately be seen by examining the definitions. For example, if at any point in the calculation, say after $\mathrm{n}$ steps, we have $\neg$ [INIT $\Rightarrow\langle\forall \mathrm{i}$ : $0 \leq \mathrm{i}<\mathrm{n}:: \mathrm{g}$. True $\rangle]$ then we can immediately conclude that $\mathrm{I}$ is not an invariant of the program and stop the calculation. Also, one can replace $g^{\mathbf{i}}$.True in any step with a stronger (more manageable) predicate and conclude from the monotonicity of WLP that the calculated predicate $K$ implies wst.I. Thus, for such a $K$, (INIT $\Rightarrow \mathrm{K}) \Rightarrow$ invariant' I.

Definition (ensures')

$$
\begin{aligned}
\text { Pensures' }^{\mathrm{Q}} \equiv & \mathrm{P} \text { unless' } \mathrm{Q} \wedge \\
& \langle\exists \mathrm{s} \in \mathrm{F} . \mathrm{ASSIGN}::[\text { sst.INIT } \wedge \mathrm{P} \wedge \neg \mathrm{Q} \Rightarrow \text { wlp.s. }(\mathrm{P} \vee \mathrm{Q})]\rangle \\
& \equiv \mathrm{P} \text { unless' } \mathrm{Q} \wedge\langle\exists \mathrm{s}::[\mathrm{INIT} \Rightarrow \text { wst }(\mathrm{P} \wedge \neg \mathrm{Q} \Rightarrow \text { wlp.s. }(\mathrm{P} \vee \mathrm{Q})]\rangle
\end{aligned}
$$

\section{Definition $\left(\mapsto^{\prime}\right)$}

Three inference rules for $\mapsto$ ' are obtained from the previous ones by replacing ensures with ensures' and $\mapsto$ with $\mapsto$ '.

\subsection{Subscripted Properties}

It is desirable to define additional properties that are stronger than unless' and ensures' given above. These allow certain results to be easily proved and provide a convenient way to keep track of the weakest invariants used in the proof of properties 
of programs that may be composed with other programs or be refined using refinement mappings as described in [Sa90].

Definition (subscripted properties)

$$
\begin{aligned}
& \left(\mathrm{P} \text { unless }^{\prime} \mathrm{Q}\right)_{\mathrm{I}} \equiv[\text { sst. INIT } \Rightarrow \mathrm{I}] \wedge[\mathrm{I} \Rightarrow(\mathrm{P} \wedge \neg \mathrm{Q} \Rightarrow \text { WLP. }(\mathrm{P} \vee \mathrm{Q}))] \\
& \equiv \text { invariant }^{\prime} \mathrm{I} \wedge[\mathrm{P} \wedge \neg \mathrm{Q} \wedge \mathrm{I} \Rightarrow \mathrm{WLP} .(\mathrm{P} \vee \mathrm{Q})] \\
& \left(P \text { ensures }^{\prime} \mathrm{Q}\right)_{\mathrm{I}} \equiv(\mathrm{P} \text { unless' } \mathrm{Q})_{\mathrm{I}} \wedge\langle\exists \mathrm{s}::[\mathrm{I} \wedge \mathrm{P} \wedge \neg \mathrm{Q} \Rightarrow \text { wlp.s. }(\mathrm{P} \vee \mathrm{Q})]\rangle \\
& \equiv\left(P \text { unless } \mathrm{Q}_{\mathrm{I}} \wedge\langle\exists \mathrm{s}::[\mathrm{I} \Rightarrow \text { wst. }(\mathrm{P} \wedge \neg \mathrm{Q} \Rightarrow \text { wlp.s. }(\mathrm{P} \vee \mathrm{Q}))]\rangle\right. \\
& \left(\mathrm{P} \mapsto{ }^{\prime} \mathrm{Q}\right)_{\mathrm{I}}
\end{aligned}
$$

The three proof rules for $\left(P \mapsto{ }^{\prime} \mathrm{Q}\right)_{\text {I }}$ are obtained from those for $\mapsto^{\prime}$ by replacing every ensures' and $\mapsto$ ' with the conresponding property subscripted with $\mathrm{I}$.

Several useful facts about subscripted properties are given in the next theorem:

Theorem (subscripted properties)

In a. b. and c. below, all occurences of Prop in the statement are to be replaced with one of unless, ensures, or $\mapsto$, in d. by unless or ensures.
a. $\quad$ Prop' $Q \equiv(P \text { Prop' } Q)_{\text {Sst.INIT }}$
b. $\quad\left(P^{P}\right.$ Prop $^{\prime} \mathrm{Q}_{\mathrm{I}} \wedge$ invariant' $\mathrm{J} \Rightarrow\left(\mathrm{P} \text { Prop }^{\prime} \mathrm{Q}\right)_{\mathrm{J}} \wedge \mathrm{I}$
c. $\quad(\text { P Prop' } \mathrm{Q})_{\mathrm{I}} \Rightarrow$ P Prop' Q
d. (P Prop' Q)True corresponds to "directly provable" P Prop Q from [Mi90]

Part $\mathrm{c}$ above, which follows directly from $\mathrm{a}$ and $\mathrm{b}$, is of significant practical importance. It says that to prove P Prop' $\mathrm{Q}$ it is sufficient to prove (P Prop' Q) I for any convenient invariant' I and actually computing sst.INIT is unnecessary. In particular, True is a very convenient invariant in many cases since it holds immediately.

The standard UNITY derived rules for combining properties of a single program, for example, the conjunction theorem (see appendix), can be directly proved for subscripted properties (and thus, using the second fact, for normal properties) using both directions ( $\equiv$ ) in the definitions. This is the same approach that was used in 
[ChMi88]. However, the proofs in [ChMi88] turned out to be incomplete (fortunately, the theorems are still correct), needing the additional application of a meta-meta theorem proved in [Mi90] when the definition of unless and ensures are as given in that paper.

\section{Substitution Rule}

In this section, we give formally justified substitution rules that can replace the substitution axiom in the UNITY proof system. The statement of the substitution axiom is an informal rendering of a special case of the theorem.

Theorem (substitution)

Let $\mathrm{I}$ and $\mathrm{J}$ be predicates, $\mathrm{Z}$ and $\mathrm{Y}$ be either both predicates or both variable. $\mathrm{P}$ and $\mathrm{Q}$ map $\mathrm{Z}$ to predicates, and all occurances of Prop can be replaced by either unless, ensures, or $\mapsto$ '.

(P.Z Prop' Q.Z) invariant' $\mathrm{J}$

$[\mathrm{J} \Rightarrow(\mathrm{Z}=\mathrm{Y})]$

(P.Y Prop' Q.Y $)_{\mathrm{I} \wedge \mathrm{J}}$ (invariant' P.Z)

invariant' J

$[\mathrm{J} \Rightarrow(\mathrm{Z}=\mathrm{Y})]$

(invariant' P.Y $)_{\mathrm{I} \wedge \mathrm{J}}$

Proof:

$$
\begin{aligned}
& \text { unless' } \\
& {[\text { sst.INIT } \Rightarrow I] \wedge[I \Rightarrow(P . Z \wedge \neg Q . Z \Rightarrow \text { WLP. }(P . Z \vee Q . Z))] \wedge} \\
& \text { [sst.INIT } \Rightarrow J] \wedge[\mathrm{J} \Rightarrow(\mathrm{Z}=\mathrm{Y})] \\
& \Rightarrow \\
& \text { [sst.INIT } \Rightarrow \mathrm{I} \wedge \mathrm{J}] \wedge[\mathrm{I} \wedge \mathrm{J} \Rightarrow \\
& ((P . Z \wedge \neg Q . Z \Rightarrow W L P .(P . Z \vee Q . Z)) \wedge(Z=Y))] \\
& \Rightarrow \quad\{\text { Leibnitz Rule-substitution of equals }\} \\
& {[\text { sst.INIT } \Rightarrow \mathrm{I} \wedge \mathrm{J}] \wedge[\mathrm{I} \wedge \mathrm{J} \Rightarrow(P . Y \wedge \neg Q . Y \Rightarrow \text { WLP.(P.Y } \vee Q . Y)]} \\
& \equiv \\
& \text { (P.Y unless' Q.Y })_{\mathbf{I} \wedge} \mathrm{J}
\end{aligned}
$$




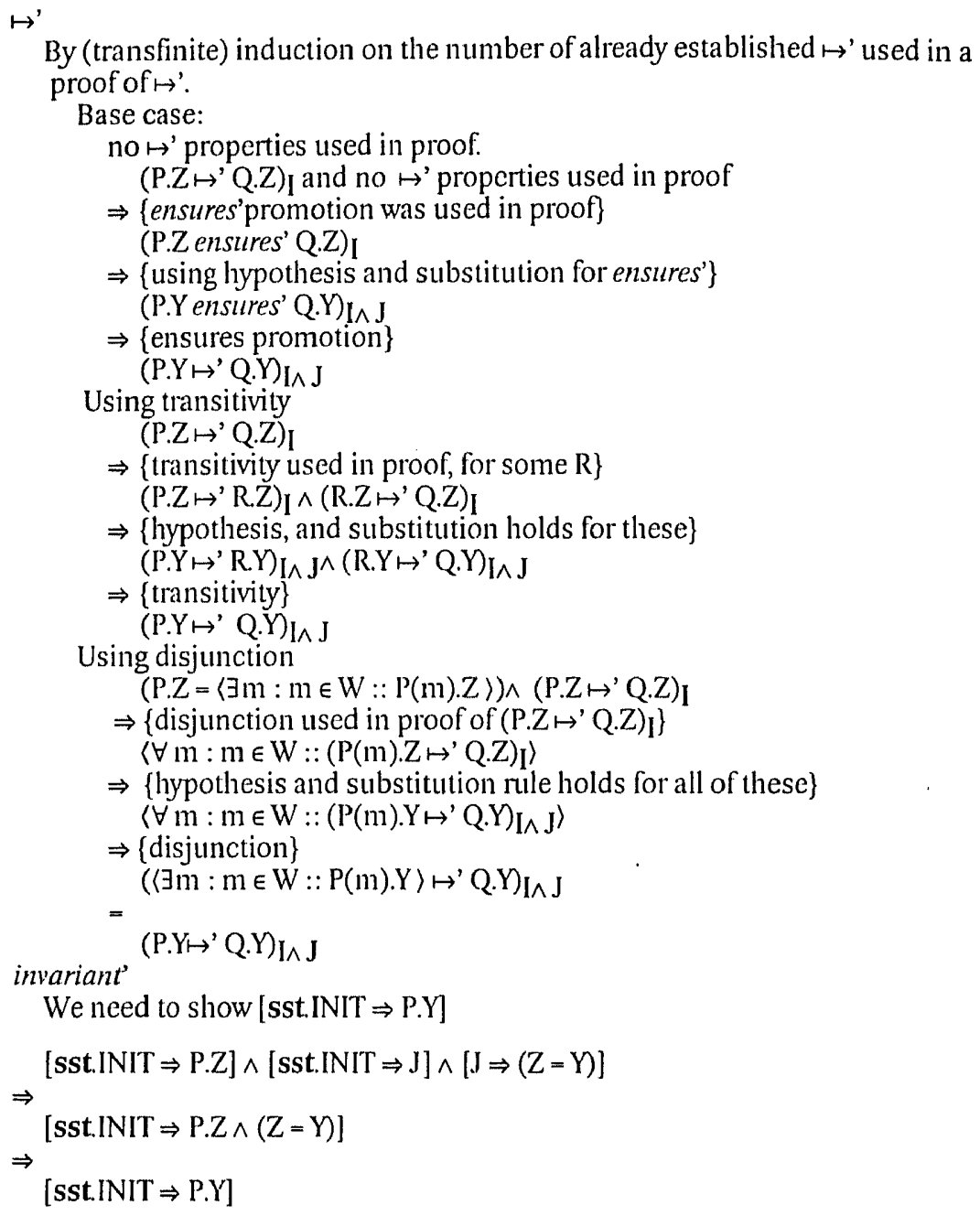

Two special cases are of particular interest: where $\mathrm{J}=\mathrm{Z}$ and $\mathrm{Y}=$ True, and where $Z=$ True and $J=Y$. We have $[Z \Rightarrow(Z=$ True $)] \equiv$ True and $[Y \Rightarrow$ (True $=Y)] \equiv$ True. These cases correspond to replacing an invariant with True, and replacing True with an invariant, respectively. 


\section{Program Composition}

In [ChMi88], the union operator for composing two programs was defined. Given two programs $F$ and $G$, a new program, the union of $F$ and $G$, denoted $F D G$ is defined as

FGG.DECLARE $\equiv$ F.DECLARE $\cup$ G.DECLARE

FLG.INIT $\equiv$ F.INIT $\wedge$ G.INIT

F]G.ASSIGN $\equiv$ F.ASSIGNu G.ASSIGN

Program union is only defined when the component programs are compatible. In particular, if the same variable name appears with different types in F.DECLARE and G.DECLARE, or if [F.INIT $\wedge$ G.INIT $=$ False], then F[G is undefined.

The union theorem 7.1 and corollaries given in [ChMi88], indicate circumstances when properties of FDG can be concluded from known properties of $F$ and $G$. For example, one part of theorem 7.1 is $P$ unless $Q$ in $F \square G=(P$ unless $Q$ in $F \wedge P$ unless $Q$ in $\mathrm{G})$ However, the compositionality there is somewhat illusory since whether or not the theorem can actually be applied depends on how the properties have been proved, i.e. with or without use of the substitution axiom. In contrast, the subscripted properties introduced in the previous section provide a notation expressive enough to give theorems that only require that the hypotheses hold, and do not depend on how they were proved.

\section{Theorem (Union')}

a. (stable')

(stable' I) $\mathrm{J}$ in $\mathrm{F}$

$(\text { stable' } \mathrm{I})_{\mathrm{K}}$ in $\mathrm{G}$

invariant' $\mathrm{J} \wedge \mathrm{K}$ in $\mathrm{F} \square \mathrm{G}$

$\left(\text { stable }^{\prime} \mathrm{I}\right)_{\wedge} \mathrm{K}$ in $\mathrm{F} \square \mathrm{G}$ b. (invariant')

(invariant' $\mathrm{I})_{\mathrm{J}}$ in $\mathrm{F}$

$\left(\text { stable }^{\prime} \mathrm{I}\right)_{\mathrm{K}}$ in $\mathrm{G}$

invariant $\mathrm{J} \wedge \mathrm{K}$ in $\mathrm{F} \square \mathrm{G}$

$\left(\text { invariant }^{\mathrm{I}}\right)_{\mathrm{J} \wedge \mathrm{K}}$ in $\mathrm{F} \square \mathrm{G}$ 
c. (unless')

( $P$ unless' $Q_{\mathrm{J}}$ in $\mathrm{F}$

$(P \text { unless' } Q)_{K}$ in $G$

invariant' $\mathrm{J} \wedge \mathrm{K}$ in $\mathrm{F} \square \mathrm{G}$

$(\mathrm{P} \text { unless' } \mathrm{Q})_{\mathrm{J} \wedge \mathrm{K}}$ in $\mathrm{F}[\mathrm{G}$ d. (ensures')

(P ensures' Q) $\mathrm{J}$ in F

$(\mathrm{P} \text { unless' } \mathrm{Q})_{\mathrm{K}}$ in $\mathrm{G}$

invariant $\mathrm{J} \wedge \mathrm{K}$ in $\mathrm{F} \mathrm{GG}$

$(P$ ensures' $Q){ }_{J \wedge} \mathrm{K}$ in $\mathrm{F} \square \mathrm{G}$

Proof:

a. $\quad(\text { stable } \mathrm{I})_{\mathrm{J}}$ in $\mathrm{F} \wedge\left(\text { stable }^{\mathrm{I}}\right)_{\mathrm{K}}$ in $\mathrm{G}$

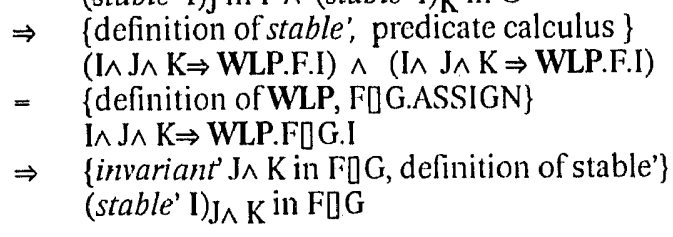

b. from a. and definition of FGG.INIT

c. $\quad(\mathrm{P} \text { unless' } \mathrm{Q})_{\mathrm{J}}$ in $\mathrm{F} \wedge(\mathrm{P} \text { unless' } \mathrm{Q})_{\mathrm{K}}$ in $\mathrm{G}$

$\Rightarrow \quad$ definition of unless', predicate calculus

$(P \wedge \neg Q \wedge J \wedge K \Rightarrow W L P . F .(P \vee Q)) \wedge(P \wedge \neg Q \wedge J \wedge K \Rightarrow$ WLP.G. $(P \vee Q))$

$=$ \{definition of WLP, F]G.ASSIGN

$P \wedge \neg Q \wedge J \wedge K \Rightarrow$ WLP.F $] G$. $(P \vee Q)$

$\Rightarrow \quad\{$ invariant $\mathrm{J} \wedge \mathrm{K}$ in $\mathrm{F} \square \mathrm{G}$, definition of unless $\}$

$\left(P \text { unless' }^{\prime} \mathrm{Q}\right)_{\mathrm{J} \wedge \mathrm{K}}$ in $\mathrm{F} \square \mathrm{G}$

d. (Pensures' $\mathrm{Q})_{\mathrm{J}}$ in $\mathrm{F} \wedge(\mathrm{P} \text { unless' } \mathrm{Q})_{\mathrm{K}}$ in $\mathrm{G}$

$\Rightarrow \quad$ definition of ensures', c. above

$(\mathrm{P} \text { unless' } \mathrm{Q})_{\mathrm{J}} \wedge \mathrm{K}$ in F[G $\wedge$

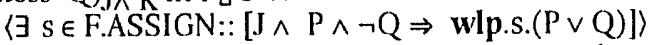

$\Rightarrow \quad$ ddefinition of FDG.ASSIGN, expand range, strengthen hypothesis

$(\mathrm{P} \text { unless' } \mathrm{Q})_{\mathrm{J} \wedge} \mathrm{K}$ in $\mathrm{F} \square \mathrm{G} \wedge$

$\langle\exists s: s \in F[G . A S S I G N::[J \wedge K \wedge P \wedge \neg Q \Rightarrow$ wlp.s. $(P \vee Q)]\rangle$

$\Rightarrow \quad\{$ invariant' $\mathrm{J} \wedge \mathrm{K}$ in $\mathrm{F}] \mathrm{G}$, definition of ensures' $\}$

(P ensures' Q) $\mathrm{J}_{\wedge} \mathrm{K}$ in $\mathrm{F} D \mathrm{G}$

At first glance these theorems seem to imply that compositionality has somehow been lost, since every result has invariant $\mathrm{J} \wedge \mathrm{K}$ in $\mathrm{FQG}$ in the hypothesis. Also, there is no result for non-subscripted properties, and indeed one can, in general, conclude nothing about sst.FuG.INIT from sst.F.INIT and sst.G.INIT. However, notice that when the subscripts Jand $\mathrm{K}$ are both True, then invariant' $\mathrm{J} \wedge \mathrm{K}$ in $\mathrm{F}$ GG follows immediately without actually having to look at statements in F[G. This will be the case in any situation where theorem 7.1 from [ChMi88] applies. 
One final point is that the theorem above is weaker than theorem 7.1 from [ChMi88] since conclusions can only be made in one direction, about the composite program given properties of the components. A theorem indicating when conclusions can be made about components is given next. Of course, the most useful case here is when $I=$ True.

\section{Theorem}

invariant $\mathrm{I}$ in $\mathrm{F}$, invariant $\mathrm{I}$ in $\mathrm{G}$, invariant $\mathrm{I}$ in $\mathrm{G}$,

a. $\quad(P \text { unless' } \mathrm{Q})_{\mathrm{I}}$ in $\left.\mathrm{F}\right] \mathrm{G} \equiv\left((\mathrm{P} \text { unless' } \mathrm{Q})_{\mathrm{I}}\right.$ in $\mathrm{F} \wedge(\mathrm{P} \text { unless' } \mathrm{Q})_{\mathrm{I}}$ in $\left.\mathrm{G}\right)$

b. (Pensures' $\mathrm{Q})_{[}$in $\mathrm{F}[\mathrm{G} \equiv$

( ( $\left(\mathrm{P}_{\text {unless' }} \mathrm{Q}_{\mathrm{I}}\right.$ in $\mathrm{F} \wedge\left(\mathrm{P}\right.$ ensures' $\mathrm{Q}_{\mathrm{I}}$ in $\left.\mathrm{G}\right)$

$\checkmark$

$\left(\left(P\right.\right.$ ensure $\mathrm{Q}_{\mathrm{I}}$ in $\mathrm{F} \wedge\left(\mathrm{P}\right.$ unless' $\mathrm{Q}_{\mathrm{I}}$ in $\left.\left.\mathrm{G}\right)\right)$

Proof:

a.

(P unless' $\mathrm{Q}_{\mathrm{I}}$ in F[G

$=$ \{definition

$(P \wedge \neg Q \wedge I \Rightarrow$ WLP.F $[\mathrm{G} .(P \vee Q)) \wedge$ invariant $\mathrm{I}$ in $\mathrm{F} \square \mathrm{G}$

$=\quad$ hypothesis, definitions of FIG.ASSIGN, WLP

$(P \wedge \neg Q \wedge I \Rightarrow$ WLP.F. $(P \vee Q) \wedge(P \wedge \neg Q \wedge I \Rightarrow$ WLP.G. $(P \vee Q))$

$=\quad$ hypothesis, definitions of unless'

$(\mathrm{P} \text { unless' } \mathrm{Q})_{I}$ in $\mathrm{F} \wedge(\mathrm{P} \text { unless' } \mathrm{Q})_{I}$ in $\left.\mathrm{G}\right)$

b. (Pensures' $\mathrm{Q}_{\mathrm{I}}$ in $\left.\mathrm{F}\right] \mathrm{G}$

$=\quad$ definition

$(\mathrm{P} \text { unless' } \mathrm{Q})_{\mathrm{I}}$ in $\mathrm{F}[\mathrm{G} \wedge$

$\langle\exists \mathrm{s}: \mathrm{s} \in \mathrm{F}]$ G.ASSIGN $::[\mathrm{I} \wedge \mathrm{P} \wedge \neg \mathrm{Q} \Rightarrow$ wlp.s. $(\mathrm{P} \vee \mathrm{Q})]\rangle$

$=\quad$ range spitting, definition of FDG.ASSIGN, part $\mathrm{a}$.

$\left(\left(\mathrm{P}\right.\right.$ unless' $\mathrm{Q}_{\mathrm{I}}$ in $\mathrm{F} \wedge\left(\mathrm{P}\right.$ unless' $\mathrm{Q}_{\mathrm{I}}$ in $\left.\mathrm{G}\right) \wedge$

( $\langle$ s: $s \in$ F.ASSIGN :: $[I \wedge P \wedge \neg Q \Rightarrow$ wlp.s. $(P \vee Q)]\rangle$

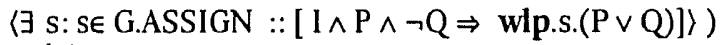

$=\quad$ \{pred.calc $\}$

$\left(\left(\mathrm{P} \text { unless }{ }^{\prime} \mathrm{Q}\right)_{\mathrm{I}}\right.$ in $\mathrm{F} \wedge\langle\exists \mathrm{s}: \mathrm{s} \in \mathrm{F} . A S S I G N::[\mathrm{I} \wedge \mathrm{P} \wedge \neg \mathrm{Q} \Rightarrow$ wlp.s. $(\mathrm{P} \vee \mathrm{Q})]\rangle$

$\wedge \quad(P \text { unless' } Q)_{\Gamma}$ in $\left.\mathrm{G}\right)$

$\checkmark$

$\left((\mathrm{P} \text { unless' } \mathrm{Q})_{\mathrm{I}}\right.$ in $\mathrm{F}$

$\wedge(P \text { unless } \mathrm{Q})_{\mathrm{I}}$ in $\mathrm{G} \wedge\langle(\exists \mathrm{s}: \mathrm{s} \in \mathrm{G} . \mathrm{ASSIGN}::[\mathrm{I} \wedge \mathrm{P} \wedge \neg \mathrm{Q} \Rightarrow$ wlp.s. $(\mathrm{P} \vee \mathrm{Q})]\rangle)$

$=$

$\left(\left(P\right.\right.$ unless' $Q_{I}$ in $F \wedge\left(P\right.$ ensures' $Q_{I}$ in $\left.G\right)$

$\left((\mathrm{P} \text { ensures' } \mathrm{Q})_{\mathrm{I}}\right.$ in $\mathrm{F} \wedge\left(\mathrm{P}\right.$ unless' $\mathrm{Q}_{\mathrm{I}}$ in $\mathrm{G}$ 


\section{On the completeness of UNITY}

Since previous work on the completeness of the UNITY proof system has not taken the substitution axiom into account, this question is addressed here. Actually, although their final conclusions do not hold, Gerth and Pnueli [GePn89] have already done most of the work. They propose a class of programs call SLP (single location programs), define semantics (as execution sequences), and give proof rules for the temporal logic operators $U$ (weak until) and o (eventually) which are known to be sound and relatively complete. Since UNITY programs are a subset of SLP programs, the inter-reducibility of proofs of $U$ and unless', and $\diamond$ and $\mapsto$ ' allows one to conclude the UNITY proof rules are sound and relatively complete. These results are straightforward and are given for the record below. Because ensures' depends on the structure of the program as well as the execution sequences, it's completeness with respect to the defined semantics is not a meaningful question, although from ensures' one can conclude $\mathrm{p}$ unless' $\mathrm{q} \wedge \mathrm{p} \mapsto{ }^{\prime} \mathrm{q}$ (which is called until in [ChMi88]). ensures' is a stronger property than until; ensures' provides the basis for $\mapsto^{\prime}$ proofs, and may also be useful in specifications since it has composibility properties which do not hold for $\mapsto$ ' [Mi90a].

\subsection{Soundness and Completeness of Unless'}

The relevant proof rule (taking some liberties with notation for consistency with this paper) in SLP is given below.

$$
\begin{aligned}
U: & {\left[Q^{\prime} \wedge P^{\prime} \Rightarrow W L P .\left(R^{\prime} \vee Q^{\prime}\right)\right] } \\
& {\left[P^{\prime} \Rightarrow W L P . P^{\prime}\right],\left[F . I N I T \Rightarrow P^{\prime}\right] } \\
& {\left[Q \wedge P^{\prime} \Rightarrow R^{\prime} \vee Q^{\prime}\right],\left[Q^{\prime} \wedge P^{\prime} \Rightarrow Q\right],\left[R^{\prime} \wedge P^{\prime} \Rightarrow R\right] }
\end{aligned}
$$

F sat $\mathrm{Q} \Rightarrow \mathrm{Q} U \mathrm{R}$

The fact that in UNITY, one can only express $\mathrm{Q} \Rightarrow \mathrm{Q} U$ R which corresponds to $\mathrm{Q}$ unless' $\mathrm{R}$, and not the more general $\mathrm{P} \Rightarrow \mathrm{Q} U \mathrm{R}$ has already been incorporated into the rule. Thus, any proof of unless' can be converted to a proof of $\mathrm{Q} \Rightarrow \mathrm{Q} U \mathrm{R}$

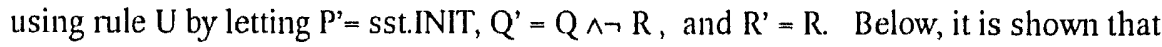
the converse holds. 
Theorem (soundness and relative completeness of unless')

unless' is sound and relatively complete

Proof:

Any proof of unless' can be converted to a proof of $\mathrm{Q} \Rightarrow \mathrm{Q} U$ R by the argument given above. Now we show any property $\mathrm{Q} \Rightarrow \mathrm{Q} U \mathrm{R}$ proved using rule $U$ can also be proved using the unless' rule. From the second line of $U$ :

(invariant $\left.\mathrm{P}^{\prime}\right)$ True. From the first line of $\mathrm{U}$ :

$Q^{\prime} \wedge P^{\prime} \wedge \neg R \Rightarrow W L P .\left(R^{\prime} \vee Q^{\prime}\right)$

$\equiv \quad\{$ lemma, stated below

$Q^{\prime} \wedge P^{\prime} \wedge \neg R \Rightarrow W L P .\left(\left(R^{\prime} \vee Q^{\prime}\right) \wedge P^{\prime}\right)$

$\Rightarrow \quad$ from $\left.U:\left(R^{\prime} \vee Q^{\prime}\right) \wedge P^{\prime} \Rightarrow Q \vee R\right)$, monotonicity of WLP)

$Q^{\prime} \wedge P^{\prime} \wedge \neg R \Rightarrow$ WLP. $\left((Q \vee R) \wedge P^{\prime}\right)$

$\Rightarrow \quad\left\{\right.$ from $\left.U: Q \wedge \neg R \wedge P^{\prime} \Rightarrow Q^{\prime} \wedge P^{\prime}\right\}$

$\mathrm{Q} \wedge \neg \mathrm{R} \wedge \mathrm{P}^{\prime} \Rightarrow$ WLP. $\left((\mathrm{Q} \vee \mathrm{R}) \vee \mathrm{P}^{\prime}\right)$

$\equiv \quad$ definition

( $\mathrm{Q}$ unless' $\mathrm{R})_{\mathrm{P}}$ '

$\Rightarrow \quad$ subscripted properties theorem

$\mathrm{Q}$ unless' $\mathrm{R}$

\section{Lemma}

$P \Rightarrow$ WLP.P

$P \wedge R \Rightarrow W L P . Q \equiv P \wedge R \Rightarrow W L P .(Q \wedge P)$

\subsection{Soundness and Completeness of $\mapsto$ '}

The relevant SLP proof rule is

$E: \quad\left[R^{\prime}(\alpha) \Rightarrow W L P .\left(R \vee\left\langle\exists \beta: \beta \leq \alpha:: R^{\prime}(\beta)\right\rangle\right)\right]$,

$\left[R^{\prime}(\alpha) \Rightarrow\right.$ wlp. $\left.f(\alpha) .\left(R \vee\left\langle\exists \beta: \beta<\alpha:: R^{\prime}(\beta)\right\rangle\right)\right]$,

[F.INIT $\left.\Rightarrow Q^{\prime}\right],\left[Q^{\prime} \Rightarrow\right.$ WLP.Q'],

$\left[Q \wedge Q^{\prime} \wedge \neg R \Rightarrow\left\langle\exists \alpha:: R^{\prime}(\alpha)\right\rangle\right]$

F sat $Q \Rightarrow \diamond R$

where $f$ is a function from the ordinals to statements in F.ASSIGN.

The UNITY property corresponding to $F$ sat $Q \Rightarrow \diamond R$ is $Q \mapsto^{\prime} R$ in $F$.

One way to prove soundness of the proof rules for $\mapsto^{\prime}$ is to show that any proof of $\mapsto$ ' can be translated into a proof of $F$ sat $Q \Rightarrow \diamond R$. This can be done by induction on the 
structure of the proofs (similar to the proof of the substitution rule for $\mapsto^{\prime}$ ), considering each proof rule separately. The work involves re-labelling predicates with appropriate ordinals, which, since there are ordinals of arbitrarily large cardinality, can always be done. However, soundness is fairly obvious and a formal proof will be omitted.

Relative completeness of the UNITY proof rules for $H^{\prime}$ are shown by reducing a proof of $F$ sat $Q \Rightarrow \diamond R$ to a proof of $Q \mapsto{ }^{\prime} R$ in $F$. The UNITY implication theorem:

$(P \Rightarrow Q) \Rightarrow(P \mapsto ' Q)$ and a special case of the cancellation theorem:

$\left(P \mapsto^{\prime} Q \vee B\right) \wedge\left(B \mapsto^{\prime} Q\right) \Rightarrow\left(P \mapsto^{\prime} Q\right)$ are used in the proof.

Theorem (soundness and relative completeness of $\mapsto^{\prime}$ )

The proof rules for $\mapsto$ ' are sound and relatively complete.

Proof:

The proof of soundness is omitted.

To prove relative completeness:

\{from the hypothesis of $E$ \}

$\left\langle\forall \alpha:: R^{\prime}(\alpha)\right.$ ensures' $R \vee\left\langle\exists \beta: \beta\left\langle\alpha:: R^{\prime}(\beta)\right\rangle\right\rangle$

$\Rightarrow$ \{ensures' promotion

$\left\langle\forall \alpha:: R^{\prime}(\alpha) \mapsto^{\prime} R \vee\left\langle\exists \beta: \beta\left\langle\alpha:: R^{\prime}(\beta)\right\rangle\right\rangle\right.$

$\Rightarrow \quad$ induction, see below

$\left\langle\forall \alpha:: R^{\prime}(\alpha) \mapsto^{\prime} R\right\rangle$

$\Rightarrow$ ddisjunction $\}$

$\left\langle\exists \alpha:: \mathrm{R}^{\prime}(\alpha)\right\rangle \mapsto^{\prime} \mathrm{R}$

$\Rightarrow$ \{hypothesis, $Q \wedge Q^{\prime} \wedge \neg R \mapsto \mapsto^{\prime}\left\langle\exists \alpha:: R^{\prime}(\alpha)\right\rangle$, transitivity\}

$\mathrm{Q} \wedge \mathrm{Q}^{\prime} \wedge \neg \mathrm{R} \mapsto{ }^{\prime} \mathrm{R}$

$\Rightarrow$ \{substitution with invariant $Q^{\prime}$, imp. th. to get $\mathrm{Q} \wedge \mathrm{R} \mapsto \mathrm{R}$, disjunction $\mathrm{Q} \mapsto{ }^{\prime} \mathrm{R}$

Induction:

base case: $R^{\prime}(0) \mapsto{ }^{\prime} R$

induction: $\left\langle\forall \beta: \beta<\alpha:: R^{\prime}(\beta) \mapsto^{\prime} R\right\rangle$

$\Rightarrow$ \{disjunction $\}$

$\left\langle\exists \beta: \beta<\alpha:: R^{\prime}(\beta)\right\rangle \mapsto^{\prime} R$

$\Rightarrow\left\{\right.$ canc. th. with $\left.R^{\prime}(\alpha) \mapsto^{\prime} \operatorname{R\vee }\left\langle\exists \beta: \beta<\alpha:: R^{\prime}(\beta)\right\rangle\right\}$

$\mathrm{R}^{\prime}(\alpha) \mapsto^{\prime} \mathrm{R}$

The above is a proof of relative completeness, which means that any unless' and ensures' properties that hold can be proved using the proof rules, provided that the underlying first order logic is strong enough to prove the hypotheses. Looking at this issue in any detail is beyond the scope of this paper, but a few remarks can be made. In proving the hypothesis, at least two issues arise. One is that the assertion language 
be strong enough to express the needed predicates. It has been shown in [Fr86] that a certain assertion language $\mathbf{L}_{\mu}$ (first order logic augmented with the least fixed point operator, constants for all recursive ordinals, an ordering relation on ordinals, and the characteristic predicate for the closing ordinal of a structure) suffices for expressing predicates needed to prove termination of fair GC programs. Thus, one can also conclude that $\mathbf{L}_{\mu}$ also suffices for $\mapsto$ ' properties of UNITY programs by noting that $P \mapsto$ ' $Q$ for a UNITY program iff a corresponding fair GC program of the form do []$_{i} Q \rightarrow S_{i}$ od terminates (the $S_{i}$ are the statements in the assign section of the program) with precondition $\mathrm{P} \wedge$ sst.INIT. Another issue is the provability of the hypotheses with a finite proof. In fact, in [ChMi88] $\mapsto$ ' is defined to hold if and only if it can be proved with a finite proof. It has been pointed out by Jan Pachl [Pa90] that this restriction to finite proofs means that some results in the literature showing the equivalence of $\mapsto$ and a certain predicate transformer are incorrect, and that a consequence of removing the restriction is that the proofs of derived properties of $\mapsto$ ' using induction on the structure of the proof (e.g. substitution theorem for $\mapsto$ ' in section 4) must formally be considered transfinite induction on the number of $\mapsto$ ' properties rather than ordinary induction on the length of the proof as is done in [ChMi88]. I have not assumed the restriction to finite proofs in this paper.

\section{Conclusion}

The work reported here was originally motivated by difficulties encountered with the substitution axiom while incorporating a technique for program transformations in UNITY [Sa90], and further inspired by a perusal of the literature which revealed that the role of the substition axiom in UNITY logic has been almost universally misunderstood. Using the strongest invariant in the definitions of unless and ensures provides theoretical advantages by clarifying the role of the initial conditions on the properties of programs, and the subscripted properties allow derived inference rules to be proved without a meta-meta-theorem and also allow union theorems to be expressed in a reasonable way. The benefits come without practical disadvantages as sufficient derived inference rules are given to allow a property of a program to be proved as easily as before.

Ackowledgements: I would like to thank Jan Pachl, Josyula Roa, Dave Naumann, Udaya Shankar, and Greg Nelson for helpful discussions and/or comments on an earlierversion. 


\section{Appendix}

Discussions of these concepts and proofs of the following well known theorems can be found, for example in [DiSch90]

Let $V$ be any bag of predicates, $f$ a predicate transformer. Then

fis universally conjunctive $\equiv[\mathrm{f} .\langle\forall \mathrm{X}: \mathrm{X} \in \mathrm{V}: \mathrm{X}\rangle \equiv\langle\forall \mathrm{X}: \mathrm{X} \in \mathrm{V}:: \mathrm{f} . \mathrm{X}\rangle]$

$f$ is universally disjunctive $\equiv[f .(\exists X: X \in V: X\rangle \equiv\langle\exists X: X \in V:: f . X\rangle]$

For the purposes of this paper, it is sufficient to know that

( $\mathrm{f}$ is universally conjunctive) $\Rightarrow$ ( $\mathrm{f}$ is and-continuous $) \wedge$ ( $\mathrm{f}$ is monotonic)

( $\mathrm{f}$ is universally disjunctive) $\Rightarrow$ ( $\mathrm{f}$ is or-continuous $) \wedge$ ( $\mathrm{f}$ is monotonic)

Theorem (Knaster-Tarski, Th 25 in [DiSch90])

For monotonic $f, Y:[f . Y \equiv Y]$ has the same strongest solution as $Y:[f . Y \Rightarrow Y]$ and has the same weakest solution as $Y:[f . Y \Leftarrow Y]$

Theorem (limit, Th 60, 60' in [DiSch90])

For or-continuous $f$, the strongest solution of $Y:[f . Y \equiv Y]$ is $\langle\exists i: 0 \leq i:: f . F a l s e\rangle$.

For and-continuous $f$, the weakest solution of $Y:[f . Y \equiv Y]$ is $\langle\forall i: 0 \leq i::$ f. True $\rangle$.

\section{References}

[ChMi88] K. Mani. Chandy and Jayadev Misra, Parallel Program Design: A Foundation, Addison-Wesley, 1988.

[DiSch90] EdsgarW. Dijkstra and Carel S. Scholten, Precicate Calculus and Program Semantics, Springer-Verlag, 1990.

[Fr86] Nissim Francez, Fairness, Springer-Verlag, 1986.

[GePn88] R. Gerth and A. Pnueli. "Rooting UNITY" Proc. Fifth Internat. Workshop on Software Specification and Design, Pittsburgh, Penn. May 19-201989..

[JKR89] C.S. Jutla, E. Knapp, J.R. Rao. "A Predicate Transformer Approach to Semantics of Parallel Programs" Proceedings of Sth Amnual ACM Symposium on Principles of Distributed Computing. 1989.

[Ke76] Robert M. Keller, "Formal Verification of Parallel Programs" CACM:19,7 July 1976, pp 371 - 384

[Kn89] E. Knapp. "A Predicate Transformer for Progress", to appear in Information Processing Letters. 
[La87] Leslie Lamport, "win and sin: Predicate Transformers for Concurrency" DEC SRC technical report \#17, May 1987.

[Liu89] Z. Liu. "A Semantic Model for UNITY" University of Warwick Computer Science Technical Report RR144, Aug. 1989.

[Mi90a] Jayadev Misra, "The Importance of Ensuring" Notes on UNITY: 11-90, January 111990.

[Mi90b] Jayadev Misra, "Soundness of the Substitution Axiom" Notes on UNITY: 14-90, March 2, 1990.

[Pa90] Jan Pachl, "Two definitions of leads-to for UNITY" draft of May 25, 1990.

[Sa90] Beverly Sanders "Stepwise Refinement of Mixed Specifications of Concurrent Programs" in M. Broy, C.B. Jones, eds. Proceedings of IFIP Working Conference on Programming Concepts and Methods, Israel, April 1990. Elsevier Science PublishersB.V. 1990.

[ShLa87] A.Udaya Shankar, Simon S. Lam, "Time-dependent Distributed Systems: proving safety, liveness and real-time properties," Distributed Computing 2, 1987 . 
Gelbe Berichte des Departements Informatlk

116 B. Sanders

117 N. Wirth

118 H.-J. Schek

H.-B Paul

M.H. Scholl

G. Weikum

119 J. Gutknecht

120 D. Mey

121 H.P. Frei

P. Schäuble

M.F. Wyle

122 P. Lăuchli

123 B. Wüthrich

124 C. Pfister

125 R. Crelier

126 A. Szyperski

127 H. Mössenböck

128 B. Sanders
Stepwise Refinement of Mixed Specifications of Concurrent Programs

Modula-2 and Object-Oriented Programming. Drawing Lines, Circles, and Ellipsis in a Raster. Flintstone.

The DASDBS Project: Objectives, Experiences, and Future Prospects

The Oberon Guide

A Predicate Calculus with Control of Derivations

The Assessment of Information Retrieval Algorithms

An Elementary Theory for Planar Graphs

Detecting Inconsistencies in Deductive Databases

The Graphics Editor Condor

The Layout System Pedro

OP2: A Portable Oberon Compiler

Network Communication in the Oberon

Environment

Coco/R: A Generator for Fast Compiler FrontEnds

Eliminating the Substitution Axiom from UNITY Logic 